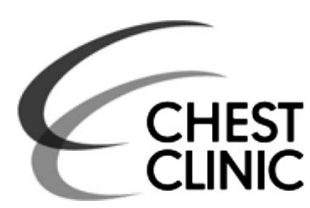

Department of Pediatric surgery, Guangzhou Women and Children's Medical Center, Affiliated Women and

Children's Medical Center of Guangzhou Medical University, Guangzhou, China

\section{Correspondence to} Dr Yan Zou, Department of Pediatric surgery, Guangzhou Women and Children's Medical Center, Affiliated Women and Children's Medical Center of Guangzhou Medical University, No: 9 Jinsui Road, Tianhe District, Guangzhou 510623, China; monknut@126.com, doctor_john82@163.com

Received 18 February 2016 Accepted 15 March 2016 Published Online First 6 April 2016

\title{
An unusual cause of shortness of breath in a young boy
}

\author{
Tianyou Yang, Jiliang Yang, Jiahao Li, Jing Pan, Yan Zou
}

A 17-month-old boy presented with shortness of breath of 3 days' duration. Physical examination showed a respiratory rate of $38 / \mathrm{min}$ and no breath sounds over the left hemithorax. Chest radiography showed a large air-filled structure in the left hemithorax, mimicking encapsulated pneumothorax (figure 1). CT identified a large structure containing

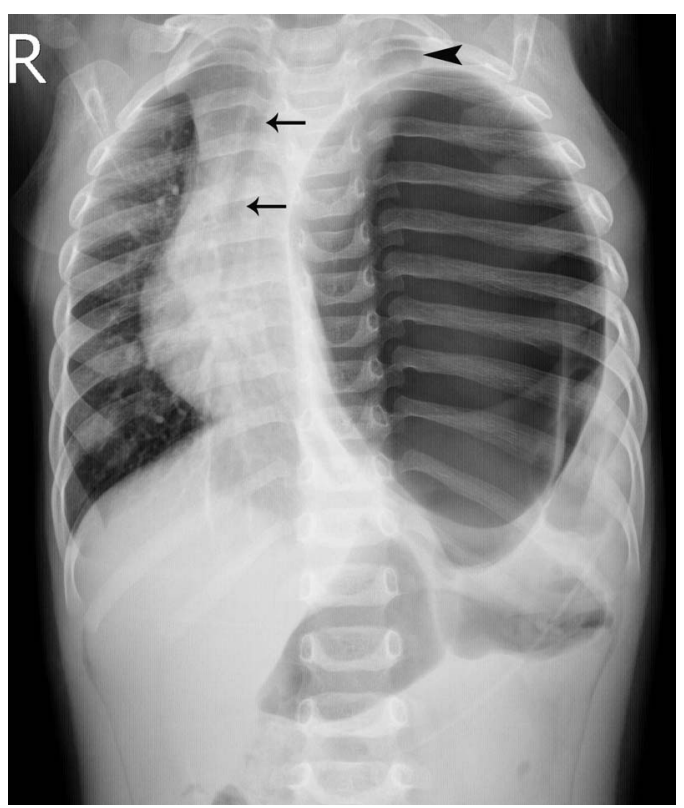

Figure 1 Chest radiography revealed a large gas-filled structure in the left hemithorax, nearly complete collapse of the left lung (the apex of the left lung, arrowhead), rightward shift of the mediastinum (arrows) and obscurity of the left hemidiaphragmatic curve. air and fluid occupying the entire left hemithorax (figure 2).

Thoracotomy revealed gastric volvulus, and the greater omentum and spleen were only visualised after needle decompression of the dilated stomach. A left Bochdalek hernia was diagnosed and the diaphragmatic defect repaired. The postoperative course was uneventful.

Late-presenting Bochdalek hernia is defined as posterolateral diaphragmatic hernia presenting beyond the neonatal period. The neonatal congenital diaphragmatic hernia is well recognised, but its presentation beyond the neonatal period varies. Respiratory and gastrointestinal symptoms are the most common clinical presentation, but cases that are asymptomatic or show an incidental finding during radiography examination are also described. ${ }^{1}$ Our patient presented with shortness of breath.

Diagnosis of a late-presenting Bochdalek hernia is challenging because of its non-specific clinical signs. Misdiagnosis can result in significant morbidity, usually due to an incorrect diagnosis of tension pneumothorax and subsequent chest-tube placement. $^{2}$ Chest radiography typically shows an airfilled structure, sometimes with an air-fluid level. A soft tissue mass may be appreciated if solid organs are herniated, and the diaphragmatic curve may be interrupted or obscured. These findings on chest radiography may mimic diaphragmatic eventration, lower lobe pneumonia or diaphragmatic mass, tension pneumothorax or pneumonia with cavitation, pleural effusion or pneumatocele. ${ }^{2}$ Upper gastrointestinal contrast study can aid a correct diagnosis, particularly when the hernia content includes hollow viscera. CT can readily identify a Bochdalek hernia, which is characterised by discontinuity of

\section{SLinked}

- http://dx.doi.org/10.1136/ thoraxjnl-2016-208590

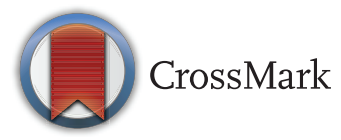

To cite: Yang T, Yang J, Li J, et al. Thorax 2016;71:772-773
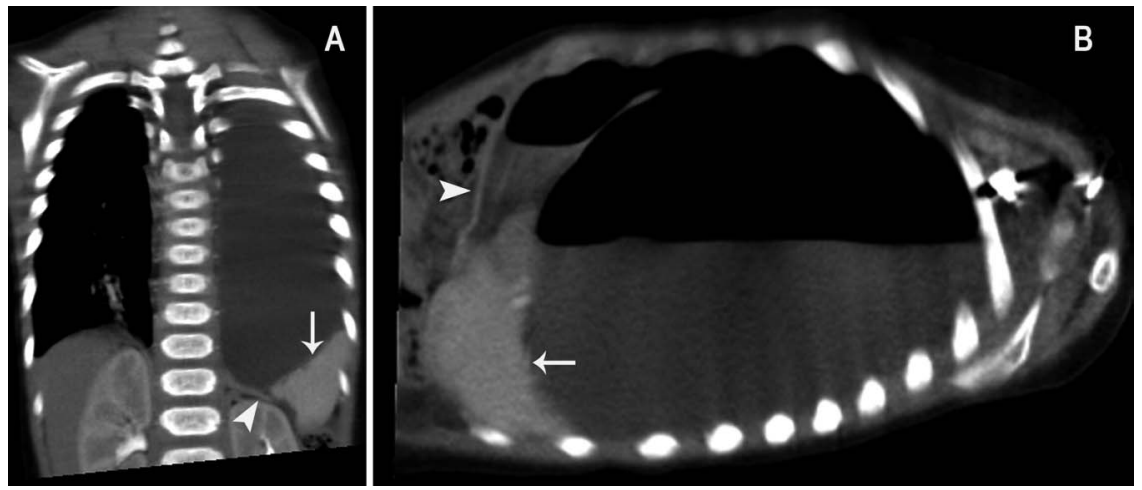

Figure 2 (A, B) CT showed a large structure containing air and fluid in the left hemithorax, discontinuity of the left hemidiaphragm (arrowhead), and protrusion of the spleen (arrow) into the thorax. 
the diaphragmatic musculature, and provide detailed information, particularly when solid organs are herniated. ${ }^{3}$

Contributors Conception and design: TY, YZ. Collection and interpretation of data: $T Y, J Y, J L, J P$. Drafting of the manuscript: TY, YZ. Approval of the final version of the manuscript: TY, JY, JL, JP, YZ.

Competing interests None declared.

Patient consent Obtained.

Ethics approval The Institutional Review Board of Guangzhou Women and Children's Medical Center (Guangzhou, China).
Provenance and peer review Not commissioned; externally peer reviewed.

\section{REFERENCES}

1 Kitano Y, Lally KP, Lally PA, Congenital Diaphragmatic Hernia Study Group. Late-presenting congenital diaphragmatic hernia. J Pediatr Surg 2005;40:1839-43.

2 Mei-Zahav M, Solomon M, Trachsel D, et al. Bochdalek diaphragmatic hernia: not only a neonatal disease. Arch Dis Child 2003;88:532-5.

3 Sandstrom CK, Stern EJ. Diaphragmatic hernias: a spectrum of radiographic appearances. Curr Probl Diagn Radiol 2011;40:95-115. 